\title{
Coleta Inteligente: Uma Proposta para Monitoramento dos Carros de Coleta de Lixo no Espaço Urbano de Cametá-PA
}

\author{
Adriano M. Borges ${ }^{1}$, Joiner S. Sá ${ }^{1}$, Davi E. S. Souza ${ }^{2}$, Diovanni M. Araújo ${ }^{1}$, \\ Fabrício S. Farias ${ }^{1}$, Diogo Acatauassu ${ }^{3}$ \\ ${ }^{1}$ Faculdade de Sistemas de Informação - Universidade Federal do Pará (UFPA) - \\ Campus Tocantins/Cametá \\ ${ }^{2}$ Faculdade de Engenharia Sanitária e Ambiental - Universidade Federal do Pará \\ (UFPA) - Campus Tucuruí \\ ${ }^{3}$ Faculdade de Computação - Universidade Federal do Pará (UFPA) - Campus \\ Castanhal \\ adrmendes10@gmail.com, fjoinersa, davisales, diovanni, fabriciosf, \\ diogoaca\} @ufpa.br
}

Abstract: The collection of solid waste in the municipality of Cametá lacks efficiency. One of the main factors is the lack of planning by the municipal government that is not building efficient policies. In this sense, this work uses technological means to improve the quality of such service. It is proposed two pieces of software, i.e., one for tracking garbage trucks and other for community use. The usability of the tools was analyzed through controlled tests and validated using criteria consolidated in the literature.

Resumo: A coleta de resíduos sólidos no município de Cametá carece de eficiência. Um dos principais fatores é a falta de planejamento por parte da prefeitura municipal que não está construindo políticas públicas eficientes. Nesse sentido, este trabalho utiliza meios tecnológicos para melhorar a qualidade de tal serviço. Desenvolvemos dois softwares para plataforma Android, um para rastrear os caminhões de lixo e outro para uso da comunidade. A usabilidade das ferramentas foi analisada através de testes controlados e validada através de critérios consolidados na literatura.

\section{INTRODUÇÃO}

O governo federal sancionou a Lei $\mathrm{n}^{\mathrm{o}} 12.305 / 2010$ que trata Política Nacional de Resíduos Sólidos (PNRS), regulamentada no mesmo ano pelo Decreto $\mathrm{n}^{\circ}$ 7.404/2010, que disciplina, entre outros tópicos, as diretrizes relativas à gestão integrada e ao gerenciamento de resíduos sólidos, tendo como norteadores a não geração de resíduos, redução, reutilização, reciclagem e tratamento dos resíduos sólidos, bem como disposição ambientalmente adequada dos rejeitos (ROCHA et al., 2017).

Mesmo com regulamentação da PNRS, a questão dos resíduos sólidos urbanos (RSU) ainda merece destaque, pelo fato de estar diretamente ligada ao crescimento e desenvolvimento das cidades e o consequente aumento vertiginoso na geração de resíduos sólidos urbanos. Um dos principais entraves está na gestão e gerenciamento 
dos RSU, que não conseguem acompanhar a quantidade de rejeitos produzidos pelas sociedades, sobretudo, na região norte do Brasil.

Segundo a Associação Brasileira de Empresas de Limpeza Pública e Resíduos Especiais (ABRELPE), estima-se que a quantidade de RSU gerados no Brasil encontrase em torno de 170 mil toneladas por dia. Na região norte esse valor gira em torno de 15.634 toneladas por dia, dos quais, aproximadamente, $19 \%$ não é coletada, confirmando a fragilidade nas políticas públicas de gestão e gerenciamento municipais, propiciando a existência de áreas clandestinas de disposição final de RSU, os chamados "lixões" (ABRELPE, 2017).

Neste cenário, capitais, estados e município têm se adequado e implantado a política da gestão de resíduos sólidos, como, Belo Horizonte, Campinas, Rio de Janeiro e São Paulo (INTERALEGIS, 2017). No norte do Brasil, o município de Cametá-PA criou a Lei $n^{\circ} 293$ de 05 de setembro de 2016 e institui o Plano Municipal de Gestão Integrada de Resíduos Sólidos, favorecendo a gestão e o gerenciamento de RSU na cidade.

Ainda assim, a cidade de Cametá-PA encontra dificuldades quando se trata de gestão e gerenciamento do RSU, especialmente no processo de coleta. No município é facilmente encontrado o acúmulo de sacolas acondicionando resíduos sólidos em ruas e calçadas, em decorrência da irregularidade do processo de coleta, provocando transtornos e prejuízos à qualidade de vida dos moradores, além de potenciais riscos de acidentes por objetos perfuro cortantes acumulados em locais inadequados e o risco de propagação de vetores.

Desta forma, com a finalidade de apresentar uma ferramenta que auxilie o processo de gestão e gerenciamento de RSU, melhorando a qualidade de vida dos cidadãos de Cametá-PA, esse trabalho tem como objetivo a apresentar o software denominado Coleta Inteligente. Este tem como finalidade dar suporte ao monitoramento dos veículos coletores de lixo do município. Para validar a proposta, este software foi avaliado via teste de usabilidade.

O restante do trabalho é organizado nas seguintes seções. A Seção apresenta os trabalhos relacionados. Já a Seção 2 apresenta a ferramenta desenvolvida nessa pesquisa. Na Seção 3 é apresentado o estudo de caso da utilização da ferramenta na cidade de Cametá-PA. Já a Seção 4 expõe os testes e resultados do trabalho. Por fim, a Seção 5 apresenta as conclusões.

\section{TRABALHOS RELACIONADOS}

Várias iniciativas têm sido realizadas com o objetivo de melhorar a coleta de resíduos sólidos. Nesta seção serão apresentadas propostas baseadas no uso da tecnologia da informação como ferramenta de apoio.

Na loja Google Play podem ser encontrados alguns softwares que corroboram com a gestão e o gerenciamento de RSU, especialmente com o processo de coleta. O software "Coleta de Resíduos" é um deles, com a finalidade de registrar o momento da coleta dos resíduos por meio de fotos, com captura automática de posicionamento (latitude/longitude), data e horário. 
Já o software "Você Recicla-Coleta de Lixo Urbana e Reciclagem" é um software para dispositivos móveis que integra informações e serviços de sistemas urbanos de limpeza, coleta e destinação de resíduos de sua cidade de forma gratuita, online e em tempo real para os habitantes.

A ferramenta "Meu Lixo", é um software em que os usuários podem ter acesso aos horários de coleta e as rotas dos caminhões em tempo real. O usuário pode ver em um mapa a localização do caminhão de coleta, ao ponto de colocar seu lixo nos pontos de coleta no período correto. Já o software "Pacscrap" permite que o usuário aprenda a separar corretamente os resíduos sólidos e informa as características do lixo que está descartando. O software "Koto City Recyclable Resource/Garbage Sorting App" é uma solução em que o usuário pode verificar as informações relacionadas aos resíduos, tais como horários de coleta de lixo, como descartar, precauções com a eliminação, uma enciclopédia de triagem e frequentes questões.

Além dos softwares, na literatura é possível encontrar diversos trabalhos que discutem soluções que dão suporte à coleta de lixo, como por exemplo os autores Asimakopoulos et al. (2016) propuseram um sistema denominado Dynacargo que tem como objetivo prever o nível de enchimento de lixeiras e gerenciar dinamicamente o procedimento de coleta de resíduos sólidos. Nesta proposta, foi utilizado um sistema embarcado com um conjunto de sensores para coletar e armazenar informações referentes ao nível dos resíduos nas lixeiras em tempo real.

No trabalho de Mavropoulos et al. (2015) foram examinados os desafios inexplorados dos softwares móveis para fornecer gerenciamento sustentável de resíduos com ênfase no desempenho de reciclagem e prevenção de resíduos, especialmente para países em desenvolvimento emergentes. Os autores apresentaram ainda, as oportunidades envolvidas no uso de softwares móveis para melhorar o desempenho de um sistema específico de gerenciamento de resíduos e o comportamento individual dos usuários.

Os autores Tapase et al. (2016) propuseram um sistema embarcado que tem como objetivo monitorar o nível de lixo das lixeiras colocadas em locais públicos evitando o transbordamento e ajudando a reduzir a poluição e diferentes riscos à saúde. Como resultado, foi apresentado um produto baseado em Arduino que monitora e envia informações sobre o nível de lixo para um dispositivo Android. Já os autores Yusof et al. (2017), propuseram um sistema de monitoramento inteligente de lixo para medir o nível de resíduos em lixeiras em tempo real e alertar os coletores via SMS quando as lixeiras estiverem cheias, para que o lixo possa ser coletado imediatamente. O sistema proposto é composto por um sensor ultrassônico para medir o nível de resíduos, um módulo GSM para enviar o SMS e um Arduino Uno que controla a operação do sistema.

Os autores De Lima et al. (2018) desenvolveram um software para auxiliar os moradores no Vale do Itajaí, com o alerta e conhecimento sobre o risco de desastre. A aplicação é desenvolvida com a plataforma Apache Cordova, as linguagens Javascript, HTML5 e CSS3, ainda foi implementado um mapa com Interface de Programação de Aplicativos do Google Maps. 
Os autores Aher et al. (2017) apresentam um sistema baseado em Internet das Coisas para informar, através de SMS, aos coordenadores de limpeza sobre o nível dos resíduos em lixeiras. O sistema é composto por uma página web onde são exibidos os status dos níveis de resíduos nas lixeiras de forma automática, desta forma é possível acessar, a qualquer momento e em qualquer lugar, os dados das lixeiras inteligentes.

Os autores Miranda et al. (2018) descreveram uma aplicação de uma arquitetura para monitorar ruídos através da coleta de dados com uso de smartphones e a visualização dos dados através de uma cidade virtual 3D.

Embora existam diversos trabalhos acadêmicos que investigam soluções para dar suporte à coleta de resíduos, estes ainda não apresentam ferramentas de monitoramento através da geolocalização dos veículos coletores. Já os softwares citados, encontrados no Google Play, apresentam soluções que auxiliam na coleta de resíduos, alguns através do compartilhamento da geolocalização dos carros de lixo. No entanto, nenhum deles dá suporte para coleta de lixo no município de Cametá.

\section{SISTEMA COLETA INTELIGENTE}

Essa seção apresenta o sistema Coleta Inteligente composto por dois softwares para a plataforma Android, que tem como função monitorar a geolocalização dos veículos que realizam a coleta de lixo da cidade de Cametá. O primeiro software denominado de Motorista tem o objetivo de enviar a localização terrestre do carro coletor de lixo para um servidor. A responsabilidade de operação do software estará com o agente responsável pelo veículo da coleta, neste caso o motorista, que irá de maneira colaborativa contribuir com o funcionamento do sistema. O segundo software denominado de Usuário, tem o objetivo de possibilitar que seus usuários acompanhem a geolocalização dos caminhões de lixo de modo on-line, visualizado no mapa da cidade, através do serviço Google Maps (2019). Esse processo dependerá da colaboração do motorista.

É importante ressaltar, que o sistema Coleta Inteligente foi desenvolvido para dispositivos com sistemas operacionais Android, pois, atualmente a plataforma contempla cerca de $85 \%$ do mercado mobile segundo o relatório realizado pela International Data Corporation (IDC, 2018).

A Figura 1 representa a arquitetura de funcionamento do sistema proposto, onde o usuário com o software Motorista recebe através do GPS sua localização e envia para o servidor as informações de latitude e longitude do carro coletor. Já os usuários que são favorecidos pela colaboração dos motoristas, os que utilizam o software Usuário, recebem do servidor a localização do veículo de modo on-line. 


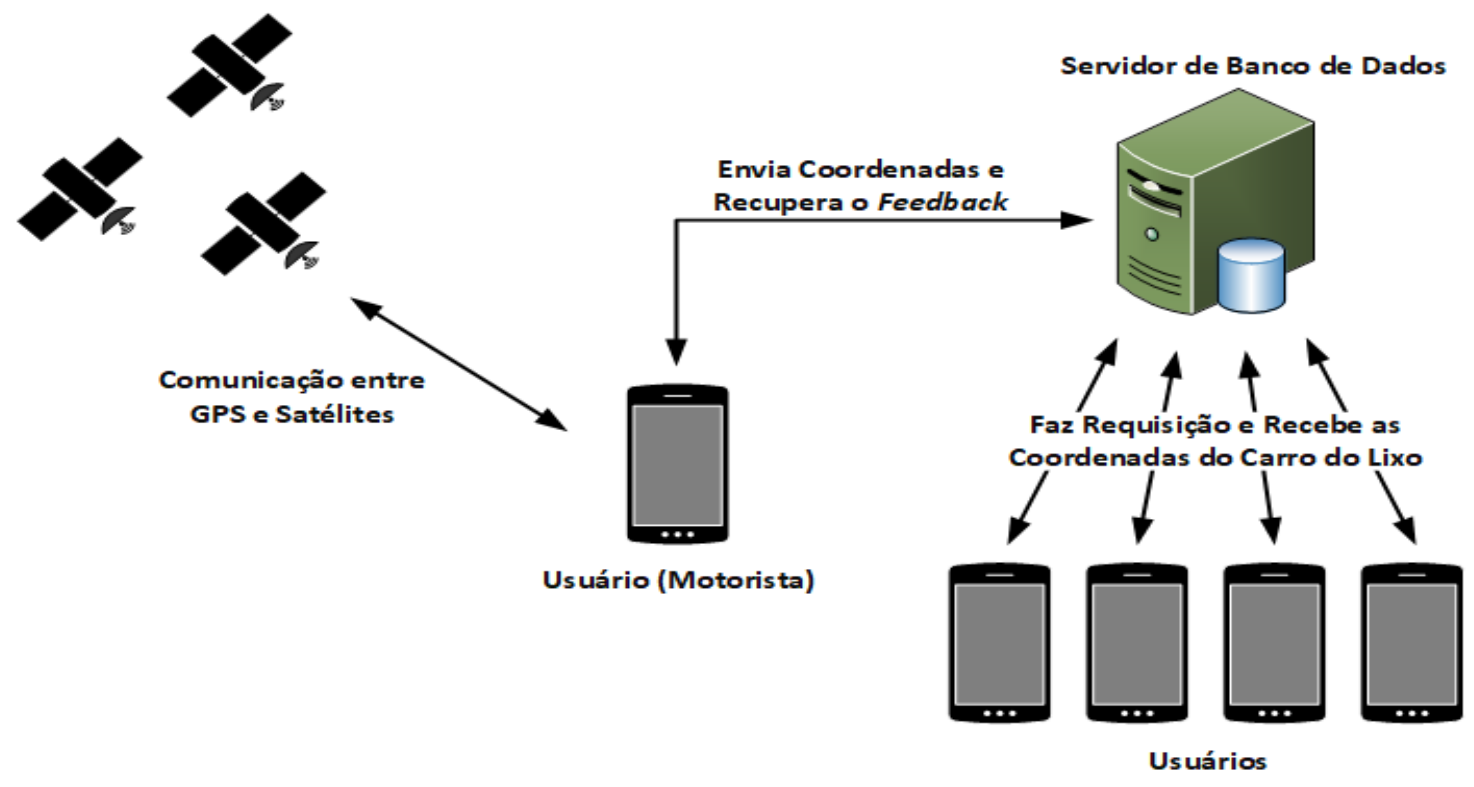

Figura 1. Arquitetura do sistema Coleta Inteligente.

A Figura 2 representa duas telas do software Motorista. Este software apresenta duas funções fundamentais para que os usuários acompanhem de forma online a coleta feita pelo veículo coletor, a primeira função é Iniciar a Coleta e a outra é Finalizar Coleta. A Figura 2 (a) ilustra a tela inicial do software, composta pelos botões que inicia e finaliza a coleta de resíduos. Já a Figura 2 (b) apresenta a tela do software que se encontra no estado de coletando lixo, essa tela apresenta para o usuário motorista o status de comunicação com o servidor.

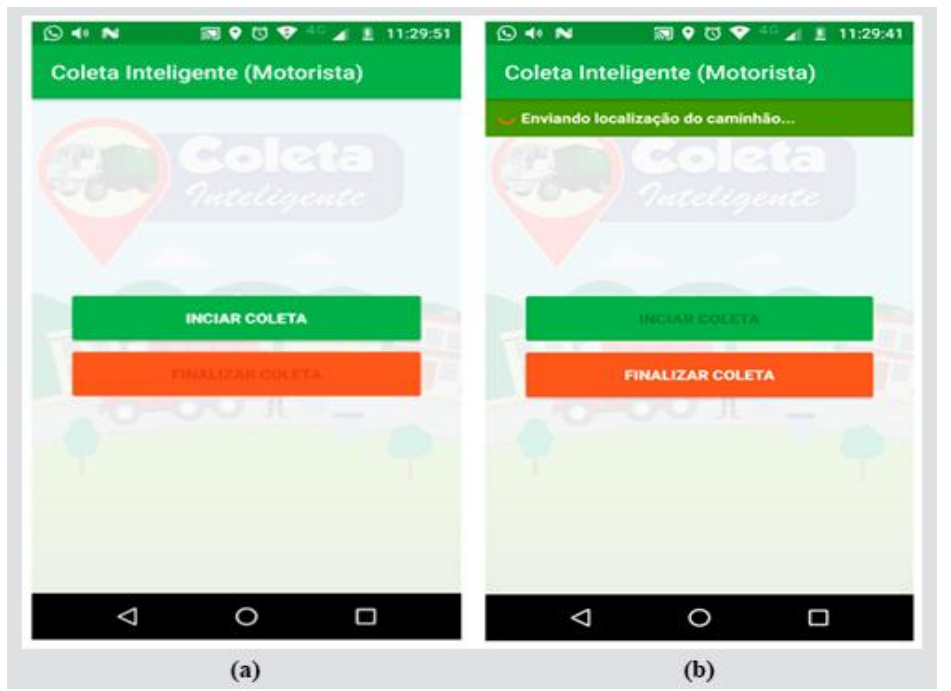

Figura 2. Telas do software Motorista. (a) Tela inicial. (b) Tela inicial no estado coletando.

O software denominado Usuário, direcionado ao público geral, é composto por três funções. A primeira é disponibilizar aos usuários a opção de acompanhar on-line os carros coletores de lixo, a segunda é possibilitar a visualização do cronograma de realização da coleta, e a última é apresentar informações e métodos de como acondicionar corretamente os resíduos até o momento da coleta. 
A Figura 3 apresenta duas das principais telas do software Usuário, onde a Figura 3 (a) ilustra a tela principal composta por três botões que dão acesso às três funções do software, e a Figura 3 (b) apresenta a tela do mapa de acompanhamento dos carros coletores de lixo, na Figura 3 (c) é apresentado ao usuário o cronograma composto por bairro, dia da semana e horário de coleta. A Figura 3 (d) ilustra a tela de informações sobre dicas e métodos de coleta de lixo.

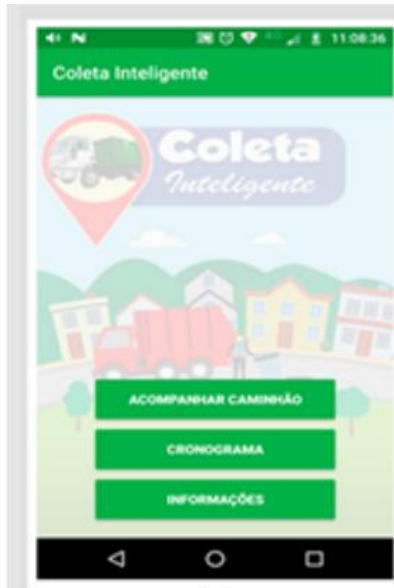

(a)

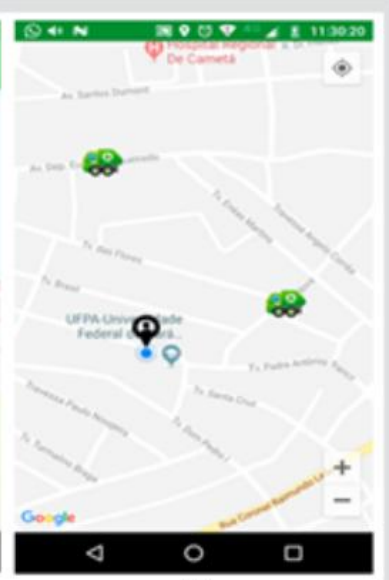

(b)

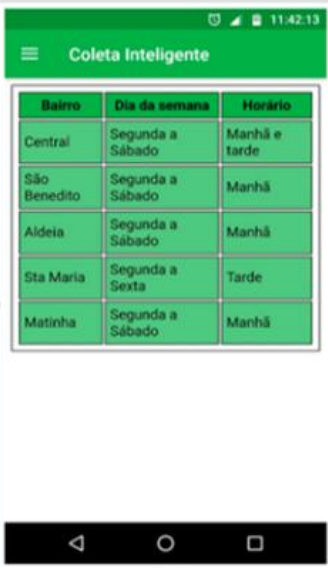

(c)

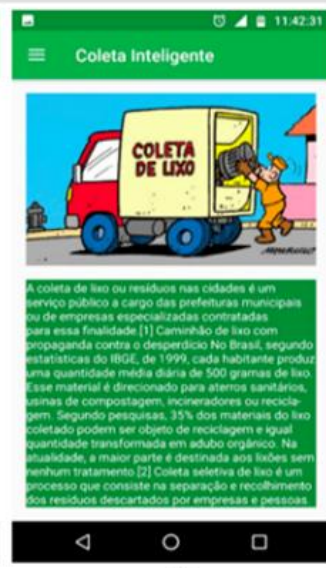

(d)

Figura 3. Telas do software Usuário. (a) Tela inicial do software. (b) Tela de acompanhamento dos carros coletores. (c) Tela de cronograma de coleta. (d) Tela de informações sobre a coleta.

\section{ESTUDO DE CASO}

Essa seção apresenta o estudo de caso da utilização do sistema Coleta Inteligente na cidade de Cametá, no estado do Pará, localizado no Brasil. Para realização dos testes, foi utilizado um veículo particular, dois smartphones, além do serviço de internet móvel de $4^{\mathrm{a}}$ geração (4G) fornecido por um provedor local. Os testes foram realizados na Rua 13 de Maio no bairro Centro. Este local foi escolhido, para os testes, devido o sinal de internet $4 \mathrm{G}$ ser melhor do que em regiões periféricas do município. Os experimentos foram realizados com 100 usuários, onde todos tinham familiaridade com smartphones e com sistemas Android.

Para realização da avaliação, foram selecionadas as atividades que podem ser frequentemente realizadas pelos usuários ao utilizar o software Usuário. Nielsen (1993) afirma que a regra básica para selecionar as atividades é que elas devem ser escolhidas para ser a mais representativa possível. Nesse caso, as atividades foram:

1. Verifique quais são as dificuldades na utilização da ferramenta;

2. Analise se o caminhão do lixo aparece no mapa no momento da coleta;

3. Verifique se usuário consegue acompanhar o cronograma da coleta;

4. Se atente se as informações são pertinentes e trazem benefícios ao usuário;

5. Comente se o acesso à internet será um fator que dificultará o processo; e 
6. Confirme se é possível implantar a ferramenta.

A avaliação deste trabalho, também foi baseada em testes de Usabilidade. A usabilidade é um dos atributos de qualidade mais importante e, segundo a norma ISO/IEC 25010, é definida como a "capacidade do produto de software de ser compreendido, aprendido, operado e atraente ao usuário, quando usado sob condições especificas".

Nos testes de usabilidade do sistema Coleta Inteligente, calculou-se o percentual de acertos através da quantidade de usuários que conseguiram realizar as atividades baseado na pesquisa de Valentim et.al (2015). Os critérios do percentual de acertos utilizados neste teste são: (a) Sucesso-Fácil: o usuário concluiu a atividade na primeira tentativa, sem problemas; (b) Sucesso- Difícil: o usuário concluiu a atividade com bastante dificuldade; e (c) Insucesso: o usuário não conseguiu completar a atividade ou desistiu. Este teste foi aplicado por dois observadores que avaliaram os 100 usuários do sistema.

\section{RESULTADOS DOS TESTES DE USABILIDADE}

Esta seção apresenta os resultados obtidos através de experimentos dos softwares que compõem o sistema Coleta Inteligente junto com a comunidade Cametaense. Os perfis de usuário são os mais diversificados possíveis, variando em idade e conhecimento do uso de smartphones. A Tabela 1 apresenta os resultados do percentual de acertos para cada atividade do experimento. Pode-se observar que a atividade que obteve o maior percentual de Insucesso (25\%) foi "Analise se o caminhão do lixo aparece no mapa no momento da coleta".

Tabela 1. Resultados dos experimentos de cada atividade.

\begin{tabular}{|c|c|c|c|}
\hline Atividades & $\begin{array}{c}\text { Sucesso } \\
\text { Fácil } \\
(\boldsymbol{\%})\end{array}$ & $\begin{array}{c}\text { Sucesso } \\
\text { Difícil } \\
(\boldsymbol{\%})\end{array}$ & $\begin{array}{c}\text { Insucesso } \\
(\mathbf{\%})\end{array}$ \\
\hline $\begin{array}{c}\text { 1- Verifique se há tantas dificuldades na } \\
\text { utilização da ferramenta. }\end{array}$ & 80 & 15 & 5 \\
\hline $\begin{array}{c}\text { 2- Analise se o caminhão do lixo aparece } \\
\text { no mapa no momento da coleta. }\end{array}$ & 65 & 10 & 25 \\
\hline $\begin{array}{c}\text { 3- Verifique se usuário consegue } \\
\text { acompanhar o cronograma da coleta. }\end{array}$ & 75 & 20 & 5 \\
\hline $\begin{array}{c}\text { 4- Se atente se as informações são } \\
\text { pertinentes e trazem benefícios ao } \\
\text { usuário. }\end{array}$ & 90 & 5 & 15 \\
\hline $\begin{array}{c}\text { 5- Comente se o acesso à internet será um } \\
\text { fator que dificultará o processo. }\end{array}$ & 80 & 5 & 5 \\
\hline $\begin{array}{c}\text { 6- Confirme se é possível implantar a } \\
\text { ferramenta. }\end{array}$ & 70 & 25 & \\
\hline
\end{tabular}

Através da avaliação da experiência do usuário pode-se observar que melhorias na aplicação devem ser realizadas a fim de que o usuário se sinta bem ao 
utilizar o software. Como exemplo, o software deve motivar, cativar e estimular os usuários mais intensamente. Além disso, os resultados do indicador Percentual de Acertos mostraram que algumas atividades foram difíceis de realizar ou não conseguiram ser realizadas. Para a identificação dessas dificuldades encontradas pelos usuários durante o uso do software, os observadores fizeram comentários no Relatório de Avaliação de Usabilidade. Os principais comentários feitos pelos observadores em relação às atividades do Teste de Usabilidade são listados na Tabela 2.

Tabela 2. Comentários dos observadores dos testes.

\begin{tabular}{|l|l|}
\hline \multicolumn{1}{|c|}{ Atividade realizada } & \multicolumn{1}{c|}{ Comentários dos Observadores (CO) } \\
\hline $\begin{array}{l}\text { Atividade 1: Verifique se há } \\
\text { tantas dificuldades na } \\
\text { utilização da ferramenta. }\end{array}$ & $\begin{array}{l}\text { CO01: o usuário precisava de mais tempo para se } \\
\text { familiarizar com ideia inovadora. }\end{array}$ \\
\hline $\begin{array}{l}\text { Atividade 2: Analise se o } \\
\text { caminhão do lixo aparece no } \\
\text { mapa no momento da coleta. }\end{array}$ & $\begin{array}{l}\text { CO02: o usuário fez a pesquisa no mapa, mas o } \\
\text { sinal de internet dificultou o processo. }\end{array}$ \\
\cline { 2 - 2 } & $\begin{array}{l}\text { CO03: o usuário achou que na parte de notificações } \\
\text { era para ser enviadas espontaneamente. }\end{array}$ \\
\hline $\begin{array}{l}\text { Atividade 3: Verifique se } \\
\text { usuário consegue acompanhar } \\
\text { o cronograma da coleta. }\end{array}$ & $\begin{array}{l}\text { CO04: o usuário realizou essa etapa com } \\
\text { facilidade. }\end{array}$ \\
\cline { 2 - 2 } $\begin{array}{l}\text { CO05: o usuário disse que não gostou da tela, mas } \\
\text { representava bem a funcionalidade que estava } \\
\text { sendo realizada. }\end{array}$ \\
\hline $\begin{array}{l}\text { Atividade 4: Se atente se as } \\
\text { informações são pertinentes e } \\
\text { trazem benefícios ao usuário. }\end{array}$ & $\begin{array}{l}\text { CO06: o usuário ficou satisfeito com a } \\
\text { simplicidade da aplicação. }\end{array}$ \\
\hline $\begin{array}{l}\text { Atividade 5: Comente se o } \\
\text { acesso à internet será um fator } \\
\text { que dificultará o processo. }\end{array}$ & $\begin{array}{l}\text { CO07: para o usuário, o acesso à internet é o fator } \\
\text { determinante para o bom funcionamento da } \\
\text { aplicação. }\end{array}$ \\
\hline $\begin{array}{l}\text { Atividade 6: Confirme se é } \\
\text { possível implantar a } \\
\text { ferramenta. }\end{array}$ & $\begin{array}{l}\text { CO08: para o usuário, a possiblidade de } \\
\text { implantação depende de alguns fatores, mas é } \\
\text { possível aplicar a ferramenta. } \\
\text { CO09: não estava claro para o usuário a opção de } \\
\text { informações educativas apresentado. }\end{array}$ \\
\hline
\end{tabular}

Os motivos pelos quais os usuários tiveram dificuldade para realizar a Atividade 2 (maior percentual de Insucesso - 25\%) são identificados através dos comentários $\mathrm{CO} 02$ e $\mathrm{CO} 03$ presentes na Tabela 4. Para o usuário seria interessante que o software notificasse com alertas. Outra atividade que obteve alto percentual de Insucesso foi a Atividade 5 (15\%). Os motivos são identificados através dos comentários $\mathrm{CO} 07$ presentes na Tabela 4. O acesso a uma internet deficiente é um fator recorrente no município de Cametá. Esse é um dos desafios que precisam ser superados. Já nas atividades 1, 3, 4 e 6, houve uma aceitação aplausível. Dentro dessas atividades o percentual de insucesso foi apenas de (5\%), identificados nos comentários CO01, CO04, CO05, CO06, CO08 e CO09 presentes na Tabela 4. O fator mais 
relevante nesse processo foi o fato de se tratar de uma ideia inovadora e de fácil utilização.

\section{CONCLUSÕES}

O presente trabalho descreveu a construção e os testes do sistema denominado Coleta Inteligente, que visa auxiliar a coleta de resíduos sólidos no município de Cametá e dar base para o processo de gestão e gerenciamento de RSU no município, bem como proporcionar melhor qualidade de vida aos moradores da cidade. O objetivo é possibilitar que o próprio usuário/cidadão acompanhe a rota dos carros coletores em cada bairro da cidade na tela de seus smartphones. Ao mesmo tempo construindo possibilidade de controle ao gestor responsável pelo serviço, de modo que o mesmo irá acompanhar a forma que está sendo executada a coleta. É importante também frisar o papel do motorista responsável pela coleta do lixo, na colaboração do serviço.

Nos resultados do teste de usabilidade, percebeu-se que 25\% dos usuários não conseguiram realizar a Atividade 2 "Analise se o caminhão do lixo aparece no mapa no momento da coleta", e $15 \%$ dos usuários não conseguiram realizar a Atividade 5 "Comente se o acesso à internet será um fator que dificultará o processo. ". Algumas das dificuldades foram relatadas pelos usuários nas entrevistas. No entanto, muitos deles afirmaram que: é fácil realizar algumas atividades, pois o software é bastante simples e intuitivo. A proposta ainda está em aberto para trabalhos futuros com o objetivo de acrescentar novas funcionalidade e adequar o sistema junto à sociedade.

\section{REFERÊNCIAS}

ABRELPE. "Panorama dos Resíduos Sólidos no Brasil". Associação Brasileira de Empresas de Limpeza Pública e Resíduos Especiais. São Paulo, 2017.

Aher, P., Kokane, S., Korade, S., Solanke, A. (2017) "Garbage Monitoring \& Alert System Using Iot". In International Journal Of Innovations In Engineering Research And Technology. Pp. 68-71.

Asimakopoulos, G., Christodoulou, S., Alefragis, P., Gizas, A., \& Triantafillou, V. (2016). "Dynacargo: The evaluation results of a Dynamic Waste Collection Management System based on Real-time and Forecasted Data". In Proceedings of the 20th Pan-Hellenic Conference on Informatics (p. 7). ACM.

Brasil. Lei 12.305, de 10 de março de 2010. Institui a Política Nacional de Resíduos Sólidos; altera a Lei no 9.605, de 12 de fevereiro de 1998; e dá outras providências. Disponível em: <http://www.planalto.gov.br/ccivil_03/_ato20072010/2010/lei/112305.htm> . Acesso em: 11 de dezembro de 2017.

De Lima, A. S., Schmidt, A. E. F., Nogueira, R. R., \& de Oliveira, P. C. F. (2018). "Aplicativo colaborativo para alerta de vulnerabilidade a alagamentos e enchentes no Vale do Itajaí". In $9^{\circ}$ Workshop de Computação Aplicada a Gestão do Meio Ambiente e Recursos Naturais (WCAMA_CSBC 2018) (Vol. 9). SBC. 
GOOGLE. "Maps: GPS e transporte público". Google Play: Google, 2019. Aplicativo móvel disponível em: $<$ https://play.google.com/store/apps/details?id=com.google.android.apps.maps\&hl= pt_BR>. Atualizado em: 17 de março 2019.

IDC (2018). "Worldwide Smartphone Volumes Will Remain Down in 2018 Before Returning to Growth in 2019 and Beyond, According to IDC". Disponível em: <https://goo.gl/Bw9mUf>. Acesso em: 02 de maio de 2019.

International Organization for Standardization ISO/IEC 25010 (2011). Systems and software engineering - SQuaRE - Software product Quality Requirements and Evaluation - System and Software Quality Models, 2011.

Interlegis. "Tratamento de Lixo: Legislação Municipal". Disponível em: <http://www.interlegis.gov.br/processo_legislativo/>. Acesso em: 15 agosto de 2017.

Lixo.com.br. "Legislação Sobre Gestão de Resíduos Sólidos e Limpeza Urbana".

Disponível em : <http://www.lixo.com.br/>. Acesso em: 09 março de 2018.

Mavropoulos, A., Tsakona, M., \& Anthouli, A. (2015) "Urban waste management and the mobile challenge". Waste Management \& Research, 33(4), 381-387.

Miranda, F. S., \& Stadzisz, P. C. (2018). "Uma proposta para monitorar a poluição sonora através de smartphones e uma cidade virtual 3D”. In $9^{\circ}$ Workshop de Computação Aplicada a Gestão do Meio Ambiente e Recursos Naturais (WCAMA_CSBC 2018) (Vol. 9). SBC.

Nielsen, J. (1993) "Usability Engineering”. In Morgan Kaufmann Publishers Inc. San Francisco, CA, USA.

SNIS - Sistema Nacional de Informações sobre Saneamento. Disponível em: <http://www.snis.gov.br/diagnostico-residuos-solidos>. Acesso em: 13 outubro 2017.

Tapase, R. B., Mohite, A., Kadam, T., \& Deshmukh, P. (2016) "Intelligent Monitoring System for Garbage Waste Bins Using Arduino". International Journal of Research in Engineering and Technology, 5(12), 82-84.

Valentim, N. M. C., Silva, W., \& Conte, T. (2015) "Avaliando a Experiência do Usuário e a Usabilidade de um aplicativo Web Móvel: um relato de experiência". In XVIII Congresso Ibero-Americano em Engenharia de Software, 2015, Lima. Congresso Ibero-Americano em Engenharia de Software (CIbSE 2015) (Vol. 1, pp. 788-801).

Yusof, N. M., Jidin, A. Z., \& Rahim, M. I. (2017). "Smart garbage monitoring system for waste management". In MATEC Web of Conferences (Vol. 97, p. 01098). EDP Sciences. 